\title{
IMPLICIT A POSTERIORI ERROR ESTIMATION USING PATCH RECOVERY TECHNIQUES *
}

\author{
TAMÁS L. HORVÁTH † AND FERENC IZSÁK ‡
}

\begin{abstract}
Implicit a posteriori error estimators are developed for elliptic boundary value problems. Local problems are formulated for the error and the corresponding Neumann type boundary conditions are approximated using a new family of gradient averaging procedure. The convergence properties of the implicit error estimator are discussed independently from that of the residual type error estimators, which provides a freedom in the choice of the boundary conditions. General assumptions are elaborated for the gradient averaging which define a family of implicit a posteriori error estimators. The performance and the favor of the method is demonstrated trough some numerical experiments.
\end{abstract}

Key words. a posteriori error estimation, gradient recovery, implicit methods

AMS subject classifications. 70K70, 70K99, 7008, 65P99

1. Introduction. Construction of accurate a posteriori error estimators for the finite element solution of PDE's is of great importance. Besides it provides a reliable stopping criterion for the consecutive refinements, it also gives a solid basis of adaptive finite algorithms. From this point of view, local estimates are of particular importance.

The starting point of many error estimation techniques is the residual based a posteriori error estimator, which provides an explicit formula for the error. The original idea in [5] has been generalized for several types of equations such as advectiondiffusion [23], convection-diffusion-"reaction" [24] and Maxwell equations [20]. Accordingly, explicit error estimators have been provided for nonconforming finite element methods [4] and a uniform approaches have been elaborated [11].

For the corresponding implicit a posteriori error estimators a Neumann type problem is formulated locally, using the numerical solution at hand and are solved in a certain local finite element space. In the simplest case, the boundary conditions for the local problems have been constructed with a simple averaging on element interfaces. To enforce the well posedness of the local problems or enhance the quality of the estimators, special equilibrated fluxes was defined and analyzed [6], [18] using the results for the residual based explicit error estimators. Thought it seems to be an involved approach, it pays off to compute more at this level: they provide local error bounds and are sensitive to the shape of the subdomain, or possibly to the mesh geometry. Implicit methods have been applied an analyzed for elliptic boundary value problems (see an overview in [3]) and generalized for time harmonic Maxwell equations [16].

Another family of powerful methods for a posteriori error estimation can obtained using gradient averaging techniques [10], which deliver simple and computationally cheap estimates. In another context, they are called recovery techniques, as the aim is to give an approximation to the gradient of the exact solution of the original problem

*This work was supported by the Dutch BSIK-project BRICKS and by the Hungarian Academy of Sciences through the OTKA-project K68253.

${ }^{\dagger}$ Department of Mathematics and Computational Science, Széchenyi University, H-9026 Győr, Egyetem tér 1, Hungary \& Department of Applied Analysis and Computational Mathematics, Eötvös University, H-1117 Budapest, Pázmány P. sétány 1/C, Hungary (thorvath@cs.elte.hu).

${ }^{\ddagger}$ Department of Applied Analysis and Computational Mathematics, Eötvös University, H-1117 Budapest, Pázmány sétány 1/C, Hungary \& Department of Applied Mathematics, University of Twente, P.O. Box 217, 7500 AE Enschede, The Netherlands (izsakf@cs.elte.hu) 
[26], [27]. Gradient averaging techniques [2], [3] can provide a reliable a posteriori error control even on unstructured grids [12] and one can make use of them in goal oriented error estimations [17]. The accuracy of the a posteriori error indicators can be enhanced if a superconvergent gradient recovery can be constructed [7], [8].

These two approaches are combined here: we prove that using an accurate approximation of the gradient - obtained with a feasible gradient average technique or patch recovery operator - as Neumann type boundary condition for the local problems results in a reliable implicit a posteriori error estimation. The favor of our approach is that we do not use any link to explicit estimators, which gives a freedom in the choice of the above operators. Moreover, the polynomial degree of the boundary data is related to the dergee of elements in the local problems. We could also get rid of strict assumptions for the mesh geometry such as the need of parallel meshes.

While in the literature usually the norms of $e_{h}$ and $\hat{e}_{h}$ are related, our result provides an upper bound of $e_{h}-\hat{e}_{h}$ in some norm, where $e_{h}$ and $\hat{e}_{h}$ yield the analytic error and the error estimator, respectively.

The paper is organized as follows. In section 2, we formalize the implicit a posteriori error estimation technique. We pose some general conditions for the gradient averaging which delivers appropriate boundary conditions for the local Neumann problems. In section 3, we prove that this combination provides an accurate a posteriori error estimator, where no link to explicit estimators is utilized. In section 4 , we provide gradient averaging techniques, which satisfy the conditions in section 2 with an appropriate polynomial order. In section 5 , we demonstrate by several numerical experiments the accuracy of the estimator for the local boundary conditions and the reliability and efficiency of the corresponding implicit a posteriori error estimate.

2. Preliminaries. We introduce implicit a posteriori error estimators for the finite element solution of the simple elliptic boundary value problem

$$
\begin{aligned}
\Delta u-k^{2} u=f & & \text { in } \Omega \\
u=g & & \text { on } \partial \Omega,
\end{aligned}
$$

where $\Omega \subset \mathbb{R}^{N}, N=2,3$ denotes a bounded polyhedral domain with $f \in H^{-1}(\Omega)$, $g \in H^{\frac{-1}{2}}(\partial \Omega)$ and $k \in \mathbb{R}^{+}$given.

We use the notations $W_{p}^{s}(\Omega)$ and $H^{s}(\Omega)$ for the Sobolev spaces on $\Omega$, where $p \in[1, \infty)$ and $s \in \mathbb{R}$, see [1], [19]. The Sobolev norm in $H^{s}(\Omega)$ is denoted with $\|\cdot\|_{s}$ and for an arbitrary subdomain $K \subset \Omega$ or a manifold $M$ we use the notations $\|\cdot\|_{s, K}$ and $\|\cdot\|_{s, M}$, respectively. In the consecutive estimates, $c_{0}$ denotes a generic constant, possibly having different values, which does not depend on the mesh size $h$.

2.1. Finite element discretization. For the finite element method a geometrically conformal triangular/tetrahedral mesh $\mathcal{T}_{h}$ of $\Omega$ is constructed [14], where $h=\max _{K \mathcal{T}_{h}} \operatorname{diam} K$ and a polynomial finite element space

$$
\mathcal{H}_{\mathbf{p}}^{h}=\left\{v_{h} \in H_{0}^{1}(\Omega):\left.v_{h}\right|_{K} \in \mathbb{P}_{p_{K}}(K), \quad \forall K \in \mathcal{T}_{h}\right\},
$$

where $\mathbb{P}_{p_{K}}(K)$ denotes the vector space of polynomials on $K$ of total degree at most $p_{K}$. We fix a finite element interpolation operator

$$
I_{h, \mathbf{p}}: W_{\infty}^{1}(\Omega) \rightarrow \mathcal{H}_{\mathbf{p}}^{h}
$$

and its restriction $I_{h, p, \hat{K}}$ to the functions supported on $\overline{\hat{K}}$, where $\hat{K}$ is a union of some finite element subdomains. 
The finite element solution of (2.1) is defined as $u_{h}=u_{h, 0}+u_{g}$, where $u_{g} \in H^{1}(\Omega)$ yields a function with $\left.u_{g}\right|_{\partial \Omega}=g$ and $u_{h, 0} \in \mathcal{H}_{\mathbf{p}}^{h}$ is the solution of the following variational problem:

$$
\left(\nabla u_{h, 0}, \nabla v_{h}\right)-k^{2}\left(u_{h, 0}, v_{h}\right)=<\Delta u_{g}-f-k^{2} u_{g}, v_{h}>\quad \forall v_{h} \in \mathcal{H}_{\mathbf{p}}^{h} .
$$

Here $(\cdot, \cdot)$ denotes the scalar product in $\left[L_{2}(\Omega)\right]^{N}, N=1,2,3$ and $<\cdot, \cdot>$ the duality pairing between $H^{-1}(\Omega)$ and $H_{0}^{1}(\Omega)$.

For the elementwise operations use the notations $u_{h, K}:=\left.u_{h}\right|_{K}$ and $u_{K}=\left.u\right|_{K}$ for the corresponding restrictions.

2.2. Implicit error estimation using patch recovery. A straightforward computation implies that for an arbitrary subdomain $K$ the restriction of the error $e_{h}=u-u_{h}$ to $K$ is the solution of the boundary value problem

$$
\left\{\begin{array}{l}
\Delta e_{h}-k^{2} e_{h}=\Delta\left(u-u_{h}\right)-k^{2}\left(u-u_{h}\right)=f-\left(\Delta u_{h}-k^{2} u_{h}\right) \quad \text { in } K \\
\partial_{\nu} e_{h}=\partial_{\nu}\left(u-u_{h}\right)=\partial_{\nu} u-\partial_{\nu} u_{h} \quad \text { on } \partial K .
\end{array}\right.
$$

The right hand side of the boundary condition is, however, in general unknown, such that we should approximate it. As a first attempt one may use a simple averaging on the common edge of two neighboring subdomains to approximate $\partial_{\nu} u$. The implicit error estimator that resulted can be related to explicit ones. This paves the way to prove its reliability and local efficiency up to the approximation of the data. For elliptic problems we refer to [3], [5], [22] and for Maxwell equations to [15], [16].

Some observations, however, motivate one to develop the above approach for the approximation of $\partial_{\nu} u$ (or, equivalently, $\partial_{\nu} e_{h}$ ) on the element interfaces.

- Using $p$ th order polynomials to solve the original problem in (2.1), the simple averaging on the interelement faces delivers a polynomial approximation for $\nabla u$ of power $p-1$. It is advised, however, that the local problems in $(2.2)$ have to be solved using a higher order finite element space than the original one. This would require a Neumann type boundary condition for the error of order $p$.

- The local problems in (2.2) could be ill posed for $k=0$ or the local error bound may lead to a crude overestimate to the error (see [3], section 6.2).

- On the other hand, in an automatic mesh refinement technique the mesh size of the neighboring elements can be highly different. Then a simple average (or even some convenient averaging techniques) does not provide an accurate approximation of the gradient.

We will construct an error estimator

$$
\hat{e}_{h}: \bigcup_{K \in \mathcal{T}_{h}} \rightarrow \mathbb{R}
$$

such that $\hat{e}_{h, K}=\left.\hat{e}_{h}\right|_{K} \in \mathbb{P}_{p_{K}+1}$ for all finite element subdomain $K \in \mathcal{T}_{h}$. Note that $\hat{e}_{h}$ is not necessarily continuous over the element interfaces. As it is usual for the local error indicators, we use the patch $\tilde{K}$ of $K$ to the construction, where

$$
\tilde{K}=\operatorname{int}\left(\bigcup_{\substack{\bar{K}_{j} \cap \bar{K}_{j} \neq \emptyset \\ K_{j} \in \mathcal{T}_{h}}} \bar{K}_{j} .\right)
$$

For a suitable approximation

$$
G_{p, K}\left(u_{h}\right) \approx \nabla u_{K}
$$


the error estimator $\hat{e}_{h, K}$ is defined as the solution of the boundary value problem

$$
\left\{\begin{array}{l}
\Delta \hat{e}_{h, K}-k^{2} \hat{e}_{h, K}=f-\Delta u_{h}+k^{2} u_{h} \quad \text { in } K \\
\partial_{\nu} \hat{e}_{h, K}=\nu \cdot G_{p, K}\left(u_{h}\right)-\partial_{\nu} u_{h} \quad \text { on } \partial K
\end{array}\right.
$$

where the right hand side is known.

Remark: The condition $k^{2}>0$ ensures that the boundary value problem in $(2.3)$ is well posed. A well-known stability estimate is recalled in Proposition 2.

2.3. Assumptions on the gradient averaging. We formulate some assumptions on the discrete gradient operator

$$
G_{p, K}: W_{\infty}^{1}(\tilde{K}) \rightarrow L_{1}(\bar{K})
$$

where $p$ yields the dependence on the local polynomial degree of the finite element space $\mathcal{H}_{\mathbf{p}}^{h}$.

While the first three are borrowed from [3], section 4, the fourth one, which streamlines the analysis at many places, is specific for our method.

(A1) $G_{p, K}(v)$ depends only on $\left.v\right|_{\tilde{K}}$.

(A2) $G_{p, K}: W_{\infty}^{1}(\tilde{K}) \rightarrow L_{1}(\bar{K})$ is continuous.

(A3) If $u \in \mathbb{P}_{p+1}(\tilde{K})$ then $G_{p, K}\left(I_{h, p, \tilde{K}} u\right)=I_{h, p, K} \nabla u_{K}$.

(A4) $G_{p, K}\left(u_{h}\right)$ is a gradient, i.e. there is a function $\mathcal{G}_{p}\left(u_{h}\right) \in W_{\infty}^{1}(\Omega)$ such that $G_{p, K}\left(u_{h}\right)=\left.\nabla \mathcal{G}_{p}\left(u_{h}\right)\right|_{\bar{K}}$

An extra condition which can imply superconvergence is the following.

(SC) There exists a constant $C(u)$ depending on $u$ such that for some $\tau \geq 0$ we have

$$
\left\|\nabla\left(u_{h}-I_{h, \mathbf{p}} u\right)\right\|_{0} \leq C(u) h^{p_{\min }+\tau}
$$

for all $h>0$, where $p_{\min }=\min _{K \in \mathcal{T}} p_{K}$.

Remarks: If $\tau=0$ then (SC) does not imply a superconvergence and the constant $C$ does not depend on $u$. This case should not be considered as an assumption, since the inequality (2.4) is a consequence of the standard finite element interpolation theory, see Chapter 4.4 in [9].

Unlike in the flux equilibration technique we do not assume that the Neumann type boundary conditions would be continuous on the element interfaces.

3. Convergence of the error estimation. In the consecutive analysis, we use the following result which can be obtained at once using a density argument.

Proposition 1. For any $w \in H^{1}(\Omega)$ we have $\Delta w \in H^{-1}(\Omega)$ and the following estimate is valid:

$$
\|\Delta w\|_{-1} \leq\|\nabla w\|_{0}
$$

We also recall a continuity estimate for elliptic boundary value problems.

Proposition 2. For an arbitrary Lipschitz domain $\Omega \subset \mathbb{R}^{n}$ with any functions $f \in H^{-1}(\Omega)$ and $g \in H^{-\frac{1}{2}}(\Omega)$ the boundary value problem

$$
\left\{\begin{array}{l}
\Delta u-k^{2} u=f \quad \text { in } \Omega \\
\partial_{\nu} u=g \text { on } \partial \Omega
\end{array}\right.
$$


has a unique solution in $H^{1}(\Omega)$ and the following estimate holds:

$$
\|u\|_{1} \leq c_{0}\|f\|_{-1}+c_{0}\|g\|_{-\frac{1}{2}, \partial \Omega}
$$

For the proof in a more general context, we refer to [19], Theorem 4.10.

The following proposition concerning the accuracy of the gradient averaging is proved in [3].

Proposition 3. Assume that the gradient averaging operator $G_{p}$ satisfies $(A 1),(A 2)$ and $(A 3)$, and also, $u \in H^{p+2}(\Omega)$ and $(S C)$ hold. Then

$$
\left\|\nabla u-G_{p}\left(u_{h}\right)\right\|_{0} \leq C(u) h^{p+\tau}|u|_{p+2}
$$

is valid, where the exponent $\tau$ is given in $(S C)$.

THEOREM 1. Assume that the conditions $(A 1),(A 2),(A 3),(A 4)$ and $(S C)$ holds. Then we have the following estimate to the precision of the error estimate:

$$
\sum_{K \in \mathcal{T}_{h}}\left\|e_{h}-\hat{e}_{h}\right\|_{1, K}^{2} \leq c_{0} \cdot C(u) h^{2(p+\tau)}|u|_{p+2}^{2}
$$

Proof: First we note that by assumption (A4) one can assume that $\mathcal{G}_{p, K}\left(u_{h}\right)$ is chosen such that $\int_{K} u-\mathcal{G}_{p, K}\left(u_{h}\right)=0$ and therefore, the Poincaré inequality implies

$$
\left\|u-\mathcal{G}_{p, K}\left(u_{h}\right)\right\|_{1, K} \leq c_{0}\left\|\nabla\left(u-\mathcal{G}_{p, K}\left(u_{h}\right)\right)\right\|_{0, K}=c_{0}\left\|\nabla u-G_{p, K}\left(u_{h}\right)\right\|_{0, K}
$$

where $c_{0}$ can be given independently of $K \subset \Omega$.

Taking the difference of $(2.2)$ and (2.3) we have that for any $K \in \mathcal{T}_{h}$

$$
\Delta\left[\left(e_{h}-\hat{e}_{h}\right)-k^{2}\left(e_{h}-\hat{e}_{h}\right)\right]=0 \quad \text { on } K,
$$

and therefore, using (2.2) and (2.3) again, for every subdomain $K \subset \Omega$ we obtain

$$
\left\{\begin{array}{l}
\Delta\left[\left(e_{h}-\hat{e}_{h}\right)-\left(u-\mathcal{G}_{p, K}\left(u_{h}\right)\right]-k^{2}\left(\left(e_{h}-\hat{e}_{h}\right)-\left(u-\mathcal{G}_{p, K}\left(u_{h}\right)\right)\right)\right. \\
=-\Delta\left(u-\mathcal{G}_{p, K}\left(u_{h}\right)\right)+k^{2}\left(u-\mathcal{G}_{p, K}\left(u_{h}\right)\right) \text { in } K \\
\partial_{\nu}\left[\left(e_{h}-\hat{e}_{h}\right)-\left(u-\mathcal{G}_{p, K}\left(u_{h}\right)\right)\right]=0 \quad \text { on } \partial K
\end{array}\right.
$$

The estimates in (3.2), Lemma 1 and (3.5) give then

$$
\begin{aligned}
& \left\|\left(e_{h}-\hat{e}_{h}\right)-\left(u-\mathcal{G}_{p, K}\left(u_{h}\right)\right)\right\|_{1, K} \\
& \quad \leq c_{0}\left\|\Delta\left(u-\mathcal{G}_{p, K}\left(u_{h}\right)\right)\right\|_{-1, K}+c_{0} \cdot k^{2}\left\|u-\mathcal{G}_{p, K}\left(u_{h}\right)\right\|_{-1, K} \\
& \quad \leq c_{0}\left\|\Delta\left(u-\mathcal{G}_{p, K}\left(u_{h}\right)\right)\right\|_{-1, K}+c_{0} \cdot k^{2}\left\|u-\mathcal{G}_{p, K}\left(u_{h}\right)\right\|_{1, K} \\
& \quad \leq c_{0}\left\|\nabla u-G_{p, K}\left(u_{h}\right)\right\|_{0, K} .
\end{aligned}
$$

Hence, the convergence result in Proposition 3 provides the estimate

$$
\begin{aligned}
& \sum_{K \in \Omega}\left\|\left(e_{h}-\hat{e}_{h}\right)-\left(u-\mathcal{G}_{p, K}\left(u_{h}\right)\right)\right\|_{1, K}^{2} \leq c_{0} \sum_{K \in \Omega}\left\|\nabla u-G_{p, K}\left(u_{h}\right)\right\|_{0, K}^{2} \\
& =c_{0}\left\|\nabla u-G_{p}\left(u_{h}\right)\right\|_{0}^{2} \leq c_{0} C^{2}(u) h^{2(p+\tau)}|u|_{p+2}^{2} .
\end{aligned}
$$


With the aid of a triangle inequality, applying (3.6), (3.5) and Proposition 3 again we conclude that

$$
\begin{aligned}
\sum_{K \subset \Omega}\left\|e_{h}-\hat{e}_{h}\right\|_{1, K}^{2} & \leq 2 \sum_{K \subset \Omega}\left\|\left(e_{h}-\hat{e}_{h}\right)-\left(u-\mathcal{G}_{p, K}\left(u_{h}\right)\right)\right\|_{1, K}^{2}+\left\|u-\mathcal{G}_{p, K}\left(u_{h}\right)\right\|_{1, K}^{2} \\
& \leq 2 \sum_{K \subset \Omega} c_{0}\left\|\nabla u-G_{p, K}\left(u_{h}\right)\right\|_{0, K}^{2}+c_{0}\left\|\nabla u-G_{p, K}\left(u_{h}\right)\right\|_{0, K}^{2} \\
& =4 c_{0}\left\|\nabla u-G_{p}\left(u_{h}\right)\right\|_{0}^{2} \\
& \leq c_{0} \cdot C(u) h^{2(p+\tau)}|u|_{p+2}^{2}
\end{aligned}
$$

as stated in the theorem.

Remark: Observe that the estimator in Theorem 1 provides not only a relation between $\sum_{K \subset \Omega}\left\|e_{h}\right\|_{1, K}^{2}$ and $\sum_{K \subset \Omega}\left\|\hat{e}_{h}\right\|_{1, K}^{2}$ but also an upper bound for the difference $\sum_{K \subset \Omega}\left\|e_{h}-\hat{e}_{h}\right\|_{1, K}^{2}$.

4. Gradient recovery using higher order fitting. We discuss in section twodimensional examples such that $\left\{\mathcal{T}_{h}\right\}$ denotes a shape regular family of geometrically conforming triangular meshes of $\Omega \subset \mathbb{R}^{2} . T \in \mathcal{T}_{h}$ denotes an arbitrary triangle for some $h$ with the vertices $E_{2}, E_{4}$ and $E_{6}$ and associated neighboring triangles $T_{1}, T_{2}$ and $T_{3}$ with the extra vertices $E_{1}, E_{3}$ and $E_{5}$, respectively, outside of $\bar{T}$, see Figure 4.1. $K$ yields a reference triangle with $\tilde{J}_{T}: K \rightarrow T$, an affine linear mapping, which is invertible and onto and has the form

$$
\tilde{J}_{T}=J_{T}+C_{T}
$$

where $C_{T}$ is constant and $J_{T}$ is linear

The mapping between a reference patch $\tilde{K}$ and $\tilde{T}$ is given by

$$
\left[J_{T}+C_{T}, J_{T_{1}}+C_{T}, J_{T_{2}}+C_{T}, J_{T_{3}}+C_{T}\right]
$$

where

$$
J_{T}\left(\bar{T} \cap \bar{T}_{j}\right)=J_{T_{j}}\left(\bar{T} \cap \bar{T}_{j}\right), j=1,2,3,
$$

i.e. they match continuously.

Before introducing gradient recovery techniques which satisfy the assumptions (A1)-(A4), we provide sufficient conditions to verify (A2).

A natural requirement for the gradient recovery is that it is transformed as the gradient by changing the coordinate system, i.e. in precise terms, for any $T \in \mathcal{T}_{<}$and $u \in L_{1}(T)$ we have

(B1) $J_{T}^{-1} \cdot\left[G_{p, K}\left(u \circ \tilde{J}_{T}\right)\right]\left(J_{T}^{-1}(x)\right)=\left[G_{p, T}(u)\right](x) \quad \forall x \in T$.

As we can not provide in general a linear or affine linear bijection between patches, an extra condition is necessary, which ensures the continuity of the gradient recovery in a sense.

(B2) Assume that for a sequence $\left(\tilde{T}_{n}\right)=\operatorname{int}\left(\bar{T}_{n} \cup \bar{T}_{n, 1} \cup \bar{T}_{n, 2} \cup \bar{T}_{n, 3}\right)$ of patches and for the corresponding mappings we have the convergence

$$
\left[J_{T_{n}}, J_{T_{n, 1}}, J_{T_{n, 2}}, J_{T_{n, 3}}\right] \rightarrow\left[J_{T}, J_{T_{1}}, J_{T_{2}}, J_{T_{3}}\right] .
$$

Then for any polynomial $u \in \mathbb{P}(\tilde{T})$ we have the convergence

$$
G_{p}\left(u_{n}\right)\left(J_{T_{n}}(x)\right) \rightarrow G_{p}(u)\left(J_{T}(x)\right), \forall x \in K,
$$

where the polynomial $u_{n} \in \mathbb{P}\left(\tilde{T}_{n}\right)$ is defined piecewise with $\left.u_{n}\right|_{T_{n, j}}=u \circ J_{T_{j}} \circ$ $J_{T_{n, j}}^{-1}, j=\emptyset, 1,2,3$. 
We point out that these three assumptions, which are easy to verify, imply $(A 2)$.

Lemma 1. Assume that (B1) and (B2) hold. Then assumption $(A 2)$ is also valid. Proof: We consider the orthogonal decomposition

$$
\mathcal{H}_{p}(\tilde{T})=\mathbf{1} \oplus \mathcal{H}_{p, 0}(\tilde{T})
$$

in the $L_{2}$-sense, where 1 denotes the subspace of constant functions in $\mathcal{H}_{p, 0}(\tilde{T})$. Note that $u \in \mathcal{H}_{p, 0}(\tilde{T})$ is equivalent with the statement $\int_{\tilde{T}} u=0$.

Since the inequality in $(A 2)$ is valid for all constant functions, it is sufficient to prove it for functions in $\mathcal{H}_{p, 0}(\tilde{T})$.

Proving by contradiction we assume that there is sequence $T^{1}, T^{2}, \ldots$ of triangles and piecewise polynomials $v_{1} \in \mathcal{H}_{p, 0}\left(\tilde{T}^{1}\right), v_{2} \in \mathcal{H}_{p, 0}\left(\tilde{T}^{2}\right), \ldots$ with $\left\|\nabla v_{j}\right\|_{L_{1}\left(\tilde{T}^{j}\right)}=1$ such that the gradient averaging is not bounded; i.e. for each positive integer $j$ we have the inequality

$$
\left\|G_{p}\left(v_{j}\right)\right\|_{L_{1}\left(T^{j}\right)} \geq j\left\|\nabla v_{j}\right\|_{L_{1}\left(\tilde{T^{j}}\right)}=j .
$$

Using (B1) we obtain the equality

$$
\left\|G_{p}\left(v_{j}\right)\right\|_{L_{1}\left(T^{j}\right)}=\operatorname{det} J_{T^{j}}^{-1} \cdot\left\|G_{p}\left(v_{j} \circ \tilde{J}_{T^{j}}\right)\right\|_{L_{1}(K)} \cdot \operatorname{det} J_{T^{j}} .
$$

and in the same way

$$
\left\|\nabla v_{j}\right\|_{L_{1}\left(\tilde{T^{j}}\right)}=\operatorname{det} J_{T^{j}}^{-1} \cdot\left\|\nabla\left(v_{j} \circ \tilde{J}_{T^{j}}\right)\right\|_{L_{1}\left(\tilde{K}_{j}\right)} \cdot \operatorname{det} J_{T^{j}},
$$

where $\tilde{K}_{j}=\tilde{J}_{T^{j}}^{-1}\left(\tilde{T}^{j}\right)$. Summarized, (4.4) and (4.5) give that

$$
\left\|G_{p}\left(w_{j}\right)\right\|_{L_{1}\left(T^{j}\right)} \geq j\left\|\nabla w_{j}\right\|_{L_{1}\left(\tilde{T^{j}}\right)}=j
$$

with $w_{j}:=v_{j} \circ \tilde{J}_{T^{j}}: \tilde{K}_{j} \rightarrow \mathbb{R}$ and the mapping between $\tilde{K}_{j}$ and $\tilde{K}$ corresponding to (4.1) is given by

$$
\left[I, J_{n, 1}, J_{n, 2}, J_{n, 3}\right]: \tilde{K}_{j} \rightarrow \tilde{K}
$$

with $I$ the identity operator. As the mesh is non-degenerate and the edges of $K$ are kept fixed, the series $\left(\left\|J_{n, j}\right\|\right)_{n}$ of the norms should be bounded and therefore, the series in (4.7) should (componentwise) converge to

$$
\left[I, J_{1}, J_{2}, J_{3}\right]: \tilde{K}^{*} \rightarrow \tilde{K}
$$

with some patch $K^{*}$ of $K$. According to the assumption (B3) for all $x \in K$ the identity

$$
G_{p}\left(w_{n}\right)(x) \rightarrow G_{p}(w)(x),
$$

where $w: K^{*} \rightarrow \mathbb{R}$ is defined with $\left.w_{n}\right|_{T_{n, j}}=w \circ J_{j} \circ J_{n, j}^{-1}$. Since $J_{j} \circ J_{n, j}^{-1} \rightarrow I$, we have

$$
\left\|G_{p}\left(w_{n}\right)\right\|_{L_{1}(K)} \rightarrow\left\|G_{p}(w)\right\|_{L_{1}(K)} \quad \text { and } \quad\left\|\nabla w_{n}\right\|_{L_{1}\left(\tilde{K}_{n}\right)} \rightarrow\|\nabla w\|_{L_{1}\left(\tilde{K}^{*}\right)}
$$

which compared with (4.6) give that

$$
\left\|G_{p}(w)\right\|_{L_{1}(K)}=\infty
$$




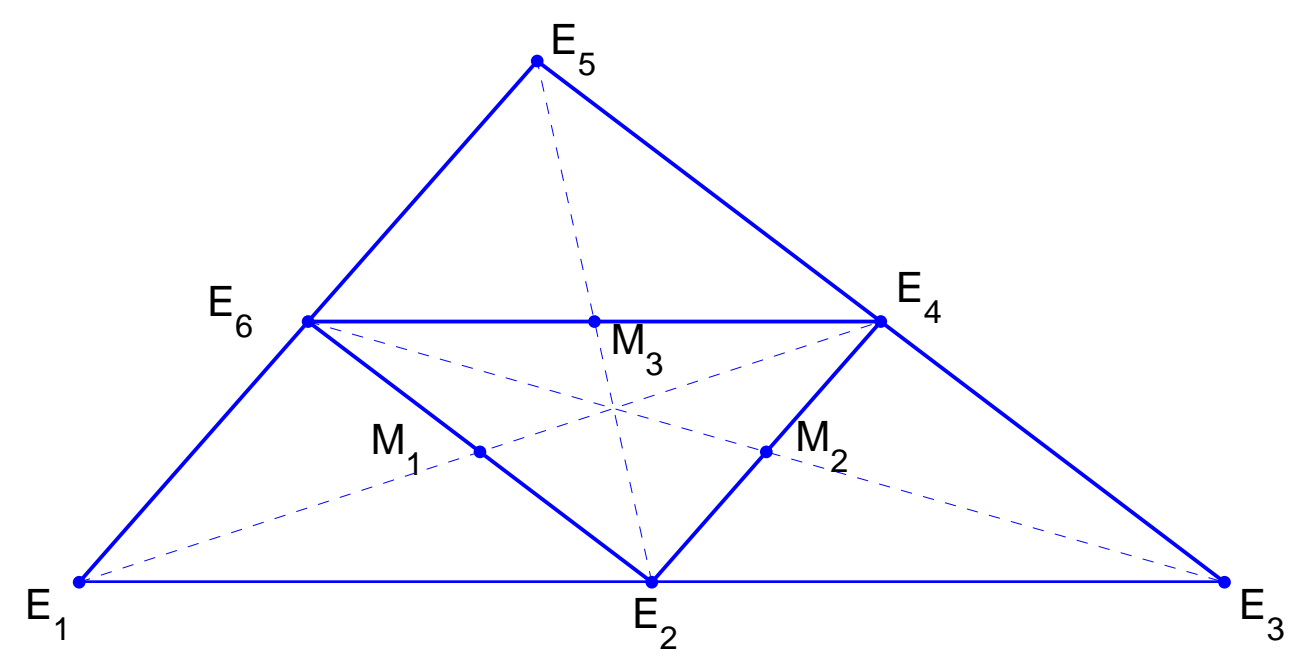

FIG. 4.1. The patch $\tilde{T}$ in a uniform tessellation with the midpoints for the first order gradient averaging.

which is a contradiction.

In general, if $\left.u_{h}\right|_{\tilde{K}} \in \mathbb{P}_{p}(\tilde{K})$, we aim to construct $G\left(u_{h}\right) \in\left[\mathbb{P}_{p}(K)\right]^{2}$ such that $G\left(u_{h}\right)$ should be a gradient of a polynomial of order $k+1$ on $\tilde{K}$.

Example 1 - Gradient recovery for $u_{h} \in \mathbf{P}_{1}(\Omega)$. The first general construction

- We fit a second order polynomial $p_{2, \tilde{T}}\left(u_{h}\right)$ to $\left\{\left(E_{i}, u_{h}\left(E_{i}\right)\right): i=1,2, \ldots, 6\right\}$.

- The first order gradient average is $G_{1, T}\left(u_{h}\right)=\left.\nabla p_{2, \tilde{T}}\left(u_{h}\right)\right|_{T}$.

Remark: A least squares fit has been applied but the particular fitting method has no importance here.

To reduce the computational costs we simplify the above process in case of a special geometry of $\hat{K}$. If $\tilde{T}$ is a triangle and consists of four uniform triangles, called uniform subdivision henceforth, then the above fitting procedure can be simplified. For this, first we determine the gradient averages in the midpoints $M_{1}, M_{2}$ and $M_{3}$ of the edges of $T$. Using the geometrical setup in Figure 4.1 we identify the vertices $E_{j}, j=1,2, \ldots, 6$ with their position vectors and introduce the notations

$$
\mathbf{v}_{i j}=\frac{E_{j}-E_{i}}{\left|E_{j}-E_{i}\right|}, i, j=1,2, \ldots, 6, i \neq j .
$$

Example 1a - Gradient recovery for $u_{h} \in \mathbb{P}_{1}(\Omega)$ on a uniform subdivision

- We define certain directional gradient averages at $M_{1}, M_{2}$ and $M_{3}$ as follows:

$$
\begin{aligned}
& \mathbf{v}_{41} \cdot G_{1, T}\left(u_{h}\right)\left(M_{1}\right)=\frac{u_{h}\left(E_{1}\right)-u_{h}\left(E_{4}\right)}{\left|E_{1}-E_{4}\right|}, \mathbf{v}_{26} \cdot G_{1, T}\left(u_{h}\right)\left(M_{1}\right)=\frac{u_{h}\left(E_{6}\right)-u_{h}\left(E_{2}\right)}{\left|E_{6}-E_{2}\right|} \\
& \mathbf{v}_{63} \cdot G_{1, T}\left(u_{h}\right)\left(M_{2}\right)=\frac{u_{h}\left(E_{3}\right)-u_{h}\left(E_{6}\right)}{\left|E_{3}-E_{6}\right|}, \mathbf{v}_{42} \cdot G_{1, T}\left(u_{h}\right)\left(M_{2}\right)=\frac{u_{h}\left(E_{2}\right)-u_{h}\left(E_{4}\right)}{\left|E_{2}-E_{4}\right|} \\
& \mathbf{v}_{25} \cdot G_{1, T}\left(u_{h}\right)\left(M_{3}\right)=\frac{u_{h}\left(E_{5}\right)-u_{h}\left(E_{2}\right)}{\left|E_{5}-E_{2}\right|}, \mathbf{v}_{64} \cdot G_{1, T}\left(u_{h}\right)\left(M_{3}\right)=\frac{u_{h}\left(E_{4}\right)-u_{h}\left(E_{6}\right)}{\left|E_{4}-E_{6}\right|} .
\end{aligned}
$$




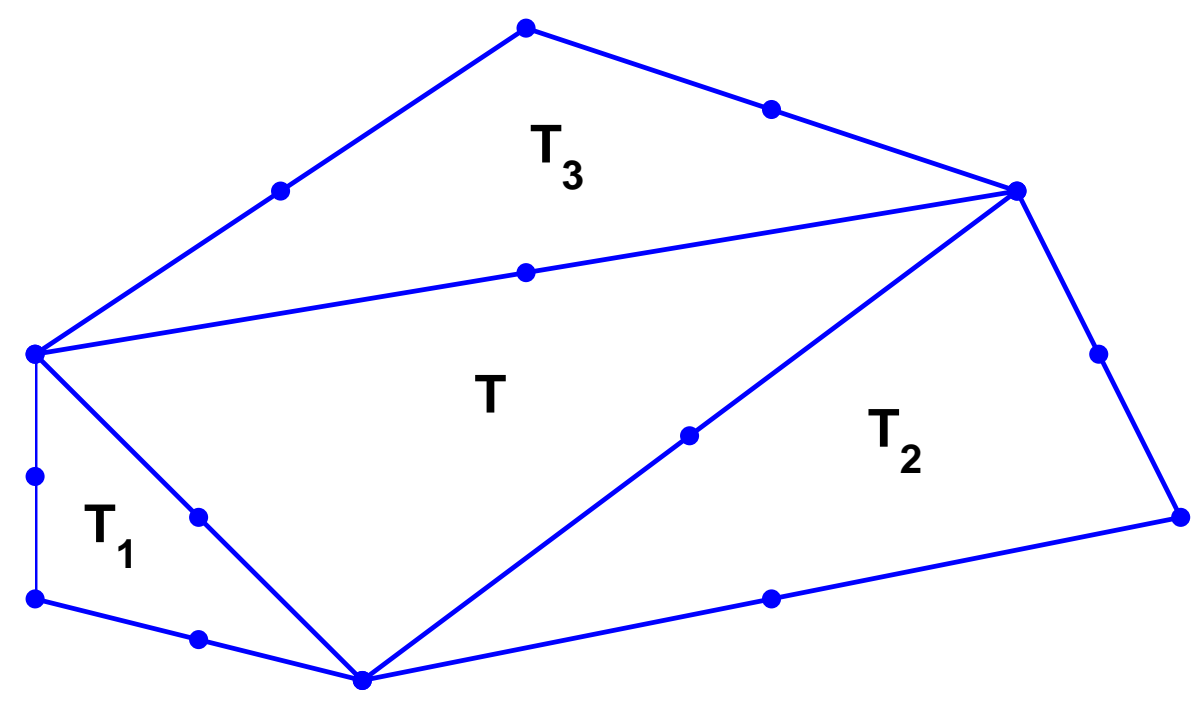

FIG. 4.2. Basis points for the second order gradient averaging.

- These determine $G_{1, T}\left(u_{h}\right)$ at $M_{1}, M_{2}$ and $M_{3}$.

- Since $G_{1, T}\left(u_{h}\right)$ is a first order polynomial in both components, it can be obtained with a linear interpolation using $G_{1, T}\left(u_{h}\right)\left(M_{1}\right), G_{1, T}\left(u_{h}\right)\left(M_{2}\right)$ and $G_{1, T}\left(u_{h}\right)\left(M_{3}\right)$.

Example 1b - Gradient recovery for $u_{h} \in \mathbb{P}_{1}(\Omega)$ on a uniform subdivision

For the following construction, we note that $\nabla u_{h}$ is piecewise constant.

- We define the gradient averages at $M_{1}, M_{2}$ and $M_{3}$ as follows:

$$
\begin{aligned}
& G_{1, T}\left(u_{h}\right)\left(M_{1}\right)=\frac{\left.\nabla u_{h}\right|_{\bar{T}_{1}}+\left.\nabla u_{h}\right|_{\bar{T}}}{2}, G_{1, T}\left(u_{h}\right)\left(M_{2}\right)=\frac{\left.\nabla u_{h}\right|_{\bar{T}_{2}}+\left.\nabla u_{h}\right|_{\bar{T}}}{2} \\
& G_{1, T}\left(u_{h}\right)\left(M_{3}\right)=\frac{\left.\nabla u_{h}\right|_{\bar{T}_{3}}+\left.\nabla u_{h}\right|_{\bar{T}}}{2} .
\end{aligned}
$$

- Since $G_{1, T}\left(u_{h}\right)$ is a first order polynomial in both components, it can be obtained with a linear interpolation using $G_{1, T}\left(u_{h}\right)\left(M_{1}\right), G_{1, T}\left(u_{h}\right)\left(M_{2}\right)$ and $G_{1, T}\left(u_{h}\right)\left(M_{3}\right)$ defined above.

Example 2 - Gradient recovery for $u_{h} \in \mathbb{P}_{2}(\Omega)$.

The second order approximation $u_{h}$ is determined by the nodal values at the vertices and the midpoints of the edges of the triangles (see [9], p. 73). These 15 nodal points in $\tilde{T}$ are depicted in Figure 4.2.

- We fit the above 15 data points with a full 3rd order polynomial in $\tilde{T}$, which is denoted with $p_{3, \tilde{T}}\left(u_{h}\right)$.

Remarks:

- The second order gradient average is $G_{2, T}\left(u_{h}\right)=\left.\nabla p_{3, \tilde{T}}\left(u_{h}\right)\right|_{\bar{T}}$.

1. It would be easier to fit the 3rd order polynomial to 10 data points. The advance of the setup in Example 2 is that the distribution of the basis points is symmetric with respect to the triangles. 
2. One can generalize the procedures in Examples 1 and 2 to provide a gradient recovery of an arbitrary order.

LEMma 2. The gradient recovery techniques in Example 1, Example 1a and Example $1 b$ are equivalent on uniform tessellations. Proof: On a uniform subdivision we can exactly fit a second order polynomial $q$ to $E_{j}, j=1,2, \ldots, 6$ with $u_{h}\left(E_{j}\right)=$ $q\left(E_{j}\right), j=1,2, \ldots, 6$. We define then $\hat{q}: \mathbb{R} \rightarrow \mathbb{R}$ by

$$
\hat{q}(\lambda)=q\left(E_{1}-\lambda\left|E_{1}-E_{4}\right| \mathbf{v}_{41}\right) .
$$

It is clear that $\hat{q}$ is second order with

$$
\hat{q}(0)=q\left(E_{1}\right), \quad \hat{q}(0.5)=q\left(M_{1}\right) \quad \text { and } \quad \hat{q}(1)=q\left(E_{4}\right) .
$$

Moreover, $\hat{q}^{\prime}(0.5)=\hat{q}(1)-\hat{q}(0)$ and therefore

$$
\begin{aligned}
\partial_{\mathbf{v}_{41}} q\left(M_{1}\right) & =-\hat{q}^{\prime}(0.5) \cdot \frac{1}{\left|E_{1}-E_{4}\right|}=-(\hat{q}(1)-\hat{q}(0)) \cdot \frac{1}{\left|E_{1}-E_{4}\right|} \\
& =\frac{q\left(E_{1}\right)-q\left(E_{4}\right)}{\left|E_{1}-E_{4}\right|}=\frac{u_{h}\left(E_{1}\right)-u_{h}\left(E_{4}\right)}{\left|E_{1}-E_{4}\right|} .
\end{aligned}
$$

A similar derivation gives that

$$
\partial_{\mathbf{v}_{26}} q\left(M_{1}\right)=\frac{q\left(E_{6}\right)-q\left(E_{2}\right)}{\left|E_{6}-E_{2}\right|}=\frac{u_{h}\left(E_{6}\right)-u_{h}\left(E_{2}\right)}{\left|E_{6}-E_{2}\right|}
$$

and in the same way

$$
\begin{aligned}
& \partial_{\mathbf{v}_{63}} q\left(M_{2}\right)=\frac{u_{h}\left(E_{3}\right)-u_{h}\left(E_{6}\right)}{\left|E_{3}-E_{6}\right|}, \partial_{\mathbf{v}_{42}} q\left(M_{2}\right)=\frac{u_{h}\left(E_{2}\right)-u_{h}\left(E_{4}\right)}{\left|E_{2}-E_{4}\right|}, \\
& \partial_{\mathbf{v}_{25}} q\left(M_{3}\right)=\frac{u_{h}\left(E_{5}\right)-u_{h}\left(E_{2}\right)}{\left|E_{5}-E_{2}\right|}, \partial_{\mathbf{v}_{64}} q\left(M_{3}\right)=\frac{u_{h}\left(E_{4}\right)-u_{h}\left(E_{6}\right)}{\left|E_{4}-E_{6}\right|} .
\end{aligned}
$$

This gives that the procedures in Example 1 and Example 1a results in the same averages at $M_{1}, M_{2}$ and $M_{3}$.

For proving that the averages in Example 1a and Example 1b are equivalent, we note that the gradient of an arbitrary function $q: T \rightarrow \mathbb{R}$ or $q: T_{1} \rightarrow \mathbb{R}$ are determined by $\partial_{\mathbf{v}_{41}} q$ and $\partial_{\mathbf{v}_{26}} q$ :

$$
\nabla q=A^{-1}\left(\partial_{\mathbf{v}_{41}} q, \partial_{\mathbf{v}_{26}} q\right)^{T}, \text { where } \quad A=\left(\begin{array}{c}
\mathbf{v}_{41}^{T} \\
\mathbf{v}_{26}^{T}
\end{array}\right) \in \mathbb{R}^{2 \times 2} .
$$

In this way, the gradient corresponding to the procedure in Example $1 \mathrm{~b}$ is

$$
\begin{aligned}
G_{1, T}\left(u_{h}\right)\left(M_{1}\right)= & \frac{1}{2}\left(\left.\nabla u_{h}\right|_{T_{1}}+\left.\nabla u_{h}\right|_{T}\right)\left(M_{1}\right) \\
= & \frac{1}{2} A^{-1}\left(\left(\left.\partial_{\mathbf{v}_{41}} u_{h}\right|_{T_{1}},\left.\partial_{\mathbf{v}_{26}} u_{h}\right|_{T_{1}}\right)^{T}+\left(\left.\partial_{\mathbf{v}_{41}} u_{h}\right|_{T},\left.\partial_{\mathbf{v}_{26}} u_{h}\right|_{T}\right)^{T}\right)\left(M_{1}\right) \\
= & A^{-1}\left(\frac{u_{h}\left(M_{1}\right)-u_{h}\left(E_{1}\right)}{\left|E_{1}-E_{4}\right|}+\frac{u_{h}\left(E_{4}\right)-u_{h}\left(M_{1}\right)}{\left|E_{1}-E_{4}\right|},\right. \\
& \left.\quad \frac{u_{h}\left(M_{1}\right)-u_{h}\left(E_{2}\right)}{\left|E_{6}-E_{2}\right|}+\frac{u_{h}\left(E_{6}\right)-u_{h}\left(M_{1}\right)}{\left|E_{6}-E_{2}\right|}\right) \\
= & A^{-1}\left(\frac{u_{h}\left(E_{4}\right)-u_{h}\left(E_{1}\right)}{\left|E_{1}-E_{4}\right|}, \frac{u_{h}\left(E_{6}\right)-u_{h}\left(E_{2}\right)}{\left|E_{6}-E_{2}\right|}\right) .
\end{aligned}
$$


This means, corresponding to (4.10), that in Example $1 \mathrm{~b}$ we obtain the same directional derivatives $\partial_{\mathbf{v}_{41}}$ and $\partial_{\mathbf{v}_{26}}$ as in Example 1a. In this way the recovered gradients in Example 1a and Example 1b the will be same, as well.

LEMmA 3. The recovered gradient $G_{1}\left(u_{h}\right)$ given in Example 1 satisfies the conditions in (A1)-(A4). Proof: By the construction $G_{1, T}(u)$ depends only on $\left.u\right|_{\tilde{T}}$.

To verify (B1) we first give $G_{1}\left(u \circ J_{T}\right)$. Observe that the fitted second order polynomial is $p_{2, T}\left(u \circ \tilde{J}_{T}\right)$, which is second order provides the same approximation at the basis points for $u \circ \tilde{J}_{T}$ as $p_{2, T} u$ at the basis points for $u$. Taking its gradient gives

$$
\begin{aligned}
\left(J_{T}^{-1}(x)\right) & =\left[\nabla\left(p_{2, \tilde{K}}\left(u \circ \tilde{J}_{T}\right)\right)\right]\left(J_{T}^{-1}(x)\right)=J_{T} \nabla p_{2, \tilde{T}}\left(u \circ \tilde{J}_{T} J_{T}^{-1}(x)\right) \\
& =J_{T} \nabla p_{2, \tilde{T}}(u(x))=J_{T} G_{1, T}(u)(x)
\end{aligned}
$$

such that (B1) is satisfied.

For the proof of (B2) we denote with $E_{n, 1}, E_{n, 2}, \ldots, E_{n, 6}$ the vertices of the patches $\tilde{T}_{n}$. If the convergence

$$
\left[J_{T_{n}}, J_{T_{n, 1}}, J_{T_{n, 2}}, J_{T_{n, 3}}\right] \rightarrow\left[J_{T}, J_{T_{1}}, J_{T_{2}}, J_{T_{3}}\right] .
$$

holds, then obviously $J_{T_{n}}(x) \rightarrow J_{T}(x)$ and $E_{n, j} \rightarrow E_{j}, j=1,2, \ldots, 6$. Also, by definition $u_{n}\left(E_{n, j}\right)=u\left(E_{j}\right), j=1,2, \ldots, 6$. Since the result of the fitting depends continuously on the input data, we obtain the convergence

$$
p_{2, T_{n}}\left(J_{T_{n}}(x)\right) \rightarrow p_{2, T}\left(J_{T}(x)\right) .
$$

Since here the range is finite dimensional, the gradients converge as well, i.e. for all $x \in K$ we have

$$
G_{p, T_{n}}\left(u_{n}\right)\left(J_{T_{n}}(x)\right)=\nabla p_{2, T_{n}}\left(u_{n} \circ J_{T_{n}}(x)\right) \rightarrow \nabla p_{2, T}\left(u \circ J_{T}(x)\right)=G_{p, T}(u)\left(J_{T}(x)\right),
$$

such that (B2) is satisfied.

Therefore, using Lemma 1 (A2) is satisfied, too.

If $u$ is a second order polynomial and we fit a second order polynomial to some of its nodal values, we certainly get $u$ itself such that $p_{2, \tilde{T}}\left(I_{1} u\right)=u$. Taking its gradient gives

$$
G_{1, T}\left(I_{1} u\right)=\left.\nabla p_{2, \tilde{T}}\left(I_{1} u\right)\right|_{T}=\left.\nabla u\right|_{\bar{T}}=\left.I_{1} u\right|_{T},
$$

which proves (A3).

Obviously the last condition (A4) is also valid: $G_{1, T}(u)$ is a gradient, as it is defined by $\left.\nabla p_{2, \tilde{T}}(u)\right|_{\bar{T}}$.

Lemma 4. The recovered gradient $G_{2}\left(u_{h}\right)$ satisfies the conditions in (A1)-(A4). Proof: By the construction $\left.G_{2}(u)\right|_{K}$ depends only on $\left.u\right|_{\tilde{K}}$.

To verify (B1) we first observe that the fitted second order polynomial is $p_{3, \tilde{T}}(u \circ$ $J_{K}$ ) which is third order and provides the same approximation for $u \circ J_{T}$ as $p_{3, T} u$ for $u$. Taking its gradient gives

$$
\begin{aligned}
\left(\tilde{J}_{T}^{-1}(x)\right) & =\left[\nabla\left(p_{3, \tilde{K}}\left(u \circ \tilde{J}_{T}\right)\right)\right]\left(J_{T}^{-1}(x)\right)=J_{T} \nabla p_{3, \tilde{T}}\left(u \circ \tilde{J}_{T} J_{T}^{-1}(x)\right) \\
& =J_{T} \nabla p_{3, \tilde{T}}(u(x))=J_{T} G_{2, T}(u)(x)
\end{aligned}
$$

such that (B1) is satisfied.

We can verify (B2) using the same arguments as in Lemma 3 such that according to Lemma 1 (A2) is also satisfied. 
If $u$ is a third order polynomial then the second order interpolation is executed based on the 15 values such that $p_{3, T}\left(I_{2} u\right)=u$. Taking its gradient gives

$$
G_{2, T}\left(I_{2} u\right)=\left.\nabla p_{3, \tilde{T}}\left(I_{2} u\right)\right|_{T}=\left.\nabla u\right|_{\bar{T}}=\left.I_{2} u\right|_{T}
$$

which proves (A3).

Obviously $G_{2, T}\left(u_{h}\right)$ is a gradient, as it is defined by $\left.\nabla p_{3, \tilde{T}}\left(u_{h}\right)\right|_{\bar{T}}$. This completes the proof that the conditions in (A1)-(A4) are valid.

Remarks: One can generalize the proof in Lemma 3 and Lemma 4 to prove that any higher order gradient recovery (corresponding to Examples 1 and 2) satisfies (A1)(A4).

A standard finite element convergence theory implies that the estimate in assumption (SC) is always satisfied with $\tau=0$, see [9], [14]. We do not verify here that it is also valid with some $\tau>0$. The related topic, superconvergence analysis has a large literature depending on the particular equations and finite element discretizations. For a detailed study of this condition for elliptic problems we refer to the monograph [25] and for some recent results to [?].

5. Numerical results. The performance of the a posteriori error estimator and the corresponding estimate for the Neumann type boundary data introduced in Section 4 will be demonstrated on three test cases indexed by $j=1,2,3$.

In each case, we investigate the finite element solution of the problem

$$
\left\{\begin{array}{l}
\Delta u_{j}-k^{2} u_{j}=f_{j} \quad \text { in } \Omega=(0,1) \times(0,1) \\
u_{j}=g_{j} \quad \text { on } \Gamma=\partial \Omega
\end{array}\right.
$$

with $k \in \mathbb{R}^{+}$is a given constant. The finite element tessellation of $\Omega$ is uniform.

For the computation of $u_{j, h}$ we have used Lagrange elements of first, second and third order on a uniform triangular mesh of $\Omega$.

The exact solution of (5.1) for $j=1,2,3$ are given as follows:

- Test case 1: $u_{1}(x, y)=\sin (2 \pi x) \sin (2 \pi y)$.

- Test case 2: $u_{2}(x, y)=1-\left(x^{2}+y^{2}\right)^{1 / 4}$.

- Test case 3: $u_{3}(x, y)=\arctan \left(60 \sqrt{(x-1.25)^{2}+(y+0.25)^{2}}-\frac{\pi}{3}\right)$.

These define the $f_{j}$ and $g_{j}$ in (5.1) for $j=1,2,3$.

The methods we compare are the following:

- Standard approximation based of interface averages (hereafter FA): on each edge we take the average $\partial_{\nu} e_{h}$ from the both sides. For further details, see [3] for elliptic problems and [16] for Maxwell equations.

- Gradient averaging (hereafter GA): we apply the standard techniques given in [3], [26].

- Gradient recovery using higher order fitting (hereafter LS) described in Section 4.

5.1. Global error estimators for the Neumann boundary data and the piecewise energy norm. In the local error estimates the only unknown term is the Neumann type boundary condition. Therefore, according to in Proposition 2 the accuracy of the error estimate depends on the quality of the estimate for these boundary conditions. Comparing our method is with some classical ones in all cases 
we measure the $L_{2}$ norm of $\partial_{\nu} \hat{e}_{h}-\partial_{\nu} e_{h}$ on the boundary of the subdomains:

$$
d\left(L_{2}\right):=\left(\sum_{\substack{K \subset \Omega \\ \partial K \cap \partial \Omega=\emptyset}}\left\|\partial_{\nu} e_{h}-\partial_{\nu} \hat{e}_{h}\right\|_{L_{2}(\partial K)}^{2}\right)^{\frac{1}{2}} .
$$

We also relate the local errors on the subdomains: the exact error $e_{h}$ on $K$ is computed by using the exact boundary condition $\partial_{\nu} e_{h}$ on $\partial K$, while for the implicit error estimation $\left.\hat{e}_{h}\right|_{K}$ we have used $\left.\partial_{\nu} e_{h}\right|_{\partial K}$, which has been computed with different approximations. We compute the total amount of these errors over all of the interior subdomains

$$
d\left(H^{1}\right):=\left(\sum_{\substack{K \subset \Omega \\ \partial K \cap \partial \Omega=\emptyset}}\left\|e_{h}-\hat{e}_{h}\right\|_{H^{1}(K)}^{2}\right)^{\frac{1}{2}} .
$$

The results for $u_{h} \in \mathbb{P}_{1}, \mathbb{P}_{2}$ and $\mathbb{P}_{3}$, i.e. using first, second and third order Lagrange elements are shown in Table 5.1, 5.2 and 5.3, respectively.

While in the quality of the Neumann boundary conditions no significant differences can be detected, the performance of the method LS, proposed here seems to be substantially better than the classical ones FA and GA for the piecewise energy norm. The only exception is Test case 3. Here the large oscillations in the higher order approximation of steep gradients can be the estimator for the local boundary conditions rather inaccurate, which result in an unsharp error estimators in each cases. This could be avoided by using a local mesh refinement in this critical region.

TABLE 5.1

Accuracy of the local estimations for the Neumann type boundary condition and for the local errors, when the approximations $u_{h}$ of $u_{1}, u_{2}$ and $u_{3}$, respectively, have been computed using first order Lagrange elements. The quantities in (5.2) (left) and (5.3) (right) are given for each test case using different methods.

\begin{tabular}{|c|c|c|c|}
\hline$u_{1}, d\left(L_{2}\right)$ & FA & GA & LS \\
\hline$n=5$ & 18.9076 & 14.0775 & 18.5386 \\
\hline$n=10$ & 20.2392 & 19.4027 & 19.5386 \\
\hline$n=15$ & 15.2591 & 13.9985 & 13.9397 \\
\hline
\end{tabular}

\begin{tabular}{|c|c|c|c|}
\hline$u_{1}, d\left(H_{1}\right)$ & FA & GA & LS \\
\hline$n=5$ & 18.1201 & 6.0507 & 18.1210 \\
\hline$n=10$ & 6.1978 & 6.1237 & 5.8245 \\
\hline$n=15$ & 1.7778 & 1.5945 & 1.6463 \\
\hline
\end{tabular}

\begin{tabular}{|c|c|c|c|}
\hline$u_{2}, d\left(L_{2}\right)$ & FA & GA & LS \\
\hline$n=5$ & 0.1259 & 0.0709 & 0.0560 \\
\hline$n=10$ & 0.1559 & 0.0724 & 0.0611 \\
\hline$n=15$ & 0.1646 & 0.0725 & 0.0617 \\
\hline
\end{tabular}

\begin{tabular}{|c|c|c|c|}
\hline$u_{2}, d\left(H_{1}\right)$ & $\mathrm{FA}$ & $\mathrm{GA}$ & $\mathrm{LS}$ \\
\hline$n=5$ & 0.0006 & 0.0005 & 0.0002 \\
\hline$n=10$ & 0.0005 & 0.0006 & 0.0003 \\
\hline$n=15$ & 0.0006 & 0.0008 & 0.0004 \\
\hline
\end{tabular}

\begin{tabular}{|c|c|c|c|}
\hline$u_{3}, d\left(L_{2}\right)$ & $\mathrm{FA}$ & $\mathrm{GA}$ & $\mathrm{LS}$ \\
\hline$n=5$ & 52.3118 & 44.7910 & 51.9011 \\
\hline$n=10$ & 60.8298 & 56.2916 & 62.8102 \\
\hline$n=15$ & 44.1284 & 44.8308 & 44.6453 \\
\hline
\end{tabular}

\begin{tabular}{|c|c|c|c|}
\hline$u_{3}, d\left(H_{1}\right)$ & FA & GA & LS \\
\hline$n=5$ & 89.4993 & 54.3913 & 90.2840 \\
\hline$n=10$ & 93.4484 & 78.4476 & 97.9891 \\
\hline$n=15$ & 81.3571 & 87.6624 & 81.9278 \\
\hline
\end{tabular}

5.2. Local performance of the error estimator. We present the performance of our local estimate on some subdomains shown in Figure 5.1. The graphs at the 
TABLE 5.2

Accuracy of the local estimations for the Neumann type boundary condition and for the local errors, when the approximations $u_{h}$ of $u_{1}, u_{2}$ and $u_{3}$, respectively, have been computed using second order Lagrange elements. The quantities in (5.2) (left) and (5.3) (right) are given for each test case using different methods.

\begin{tabular}{|c|c|c|c|}
\hline$u_{1}, d\left(L_{2}\right)$ & FA & GA & LS \\
\hline$n=5$ & 5.5409 & 11.9002 & 9.0425 \\
\hline$n=10$ & 5.6623 & 12.9412 & 3.5634 \\
\hline$n=15$ & 4.4471 & 11.9992 & 1.4355 \\
\hline
\end{tabular}

\begin{tabular}{|c|c|c|c|}
\hline$u_{1}, d\left(H_{1}\right)$ & FA & GA & LS \\
\hline$n=5$ & 0.7820 & 5.8598 & 1.1150 \\
\hline$n=10$ & 0.7143 & 7.2921 & 0.0714 \\
\hline$n=15$ & 0.2653 & 5.5670 & 0.0083 \\
\hline
\end{tabular}

\begin{tabular}{|c|c|c|c|}
\hline$u_{2}, d\left(L_{2}\right)$ & FA & GA & LS \\
\hline$n=5$ & 0.0354 & 0.0918 & 0.0178 \\
\hline$n=10$ & 0.0366 & 0.1099 & 0.0178 \\
\hline$n=15$ & 0.0367 & 0.1156 & 0.0178 \\
\hline
\end{tabular}

\begin{tabular}{|c|c|c|c|}
\hline$u_{2}, d\left(H_{1}\right)$ & $\mathrm{FA}$ & $\mathrm{GA}$ & $\mathrm{LS}$ \\
\hline$n=5$ & 0.0001 & 0.0004 & $<10^{-4}$ \\
\hline$n=10$ & 0.0002 & 0.0006 & $<10^{-4}$ \\
\hline$n=15$ & 0.0003 & 0.0009 & $<10^{-4}$ \\
\hline
\end{tabular}

\begin{tabular}{|c|c|c|c|}
\hline$u_{3}, d\left(L_{2}\right)$ & FA & GA & LS \\
\hline$n=5$ & 33.8184 & 29.1245 & 29.8231 \\
\hline$n=10$ & 24.0926 & 25.0117 & 24.7771 \\
\hline$n=15$ & 17.7498 & 23.1537 & 19.8181 \\
\hline
\end{tabular}

\begin{tabular}{|c|c|c|c|}
\hline$u_{3}, d\left(H_{1}\right)$ & FA & GA & LS \\
\hline$n=5$ & 55.8889 & 28.3894 & 46.5715 \\
\hline$n=10$ & 15.5860 & 33.2854 & 18.0114 \\
\hline$n=15$ & 10.0317 & 54.6338 & 15.9303 \\
\hline
\end{tabular}

TABLE 5.3

Accuracy of the local estimations for the Neumann type boundary condition and for the local errors, when the approximations $u_{h}$ of $u_{1}, u_{2}$ and $u_{3}$, respectively, have been computed using third order Lagrange elements. The quantities in (5.2) (left) and (5.3) (right) are given for each test case using different methods.

\begin{tabular}{|c|c|c|c|}
\hline$u_{1}, d\left(L_{2}\right)$ & FA & GA & LS \\
\hline$n=5$ & 4.8794 & 8.3113 & 5.1958 \\
\hline$n=10$ & 1.4455 & 7.0024 & 1.0783 \\
\hline$n=15$ & 0.4975 & 6.1275 & 0.3370 \\
\hline
\end{tabular}

\begin{tabular}{|c|c|c|c|}
\hline$u_{2}, d\left(H_{1}\right)$ & $\mathrm{FA}$ & $\mathrm{GA}$ & $\mathrm{LS}$ \\
\hline$n=5$ & 1.0356 & 1.4403 & 0.6440 \\
\hline$n=10$ & 0.0196 & 0.5068 & 0.0086 \\
\hline$n=15$ & 0.0009 & 0.3342 & 0.0006 \\
\hline
\end{tabular}

\begin{tabular}{|c|c|c|c|}
\hline$u_{2}, d\left(L_{2}\right)$ & FA & GA & LS \\
\hline$n=5$ & 0.0113 & 0.0561 & 0.0061 \\
\hline$n=10$ & 0.0114 & 0.0653 & 0.0061 \\
\hline$n=15$ & 0.0115 & 0.0679 & 0.0061 \\
\hline
\end{tabular}

\begin{tabular}{|c|c|c|c|}
\hline$u_{2}, d\left(H_{1}\right)$ & $\mathrm{FA}$ & $\mathrm{GA}$ & $\mathrm{LS}$ \\
\hline$n=5$ & $<10^{-4}$ & 0.0001 & $<10^{-4}$ \\
\hline$n=10$ & $<10^{-4}$ & 0.0001 & $<10^{-4}$ \\
\hline$n=15$ & $<10^{-4}$ & 0.0001 & $<10^{-4}$ \\
\hline
\end{tabular}

\begin{tabular}{|c|c|c|c|}
\hline$u_{3}, d\left(L_{2}\right)$ & FA & GA & LS \\
\hline$n=5$ & 34.2449 & 33.5215 & 25.6820 \\
\hline$n=10$ & 16.087 & 17.3896 & 19.2871 \\
\hline$n=15$ & 10.9046 & 12.5226 & 14.9013 \\
\hline
\end{tabular}

\begin{tabular}{|c|c|c|c|}
\hline$u_{3}, d\left(H_{1}\right)$ & $\mathrm{FA}$ & $\mathrm{GA}$ & $\mathrm{LS}$ \\
\hline$n=5$ & 66.0944 & 54.5199 & 22.3025 \\
\hline$n=10$ & 9.2497 & 7.5523 & 5.5573 \\
\hline$n=15$ & 6.1932 & 4.2965 & 7.7984 \\
\hline
\end{tabular}

left and the right hand side of Figures $5.2-5.4$ exhibit the $L_{2}$ error in the Neumann boundary data

$$
d\left(L_{2, K}\right):=\left(\left\|\partial_{\nu} e_{h}-\partial_{\nu} \hat{e}_{h}\right\|_{L_{2}(\partial K)}^{2}\right)^{\frac{1}{2}}
$$

and the $H^{1}$ error of the implicit error estimation

$$
\left(\left\|e_{h}-\hat{e}_{h}\right\|_{H^{1}(K)}^{2}\right)^{\frac{1}{2}}
$$

respectively, on the subdomains in Figure 5.1. 
One can observe that the gradient recovery operator LS proposed here delivers significantly sharper result than the classical techniques FA and GA. Another advance of this estimator is that it becomes even sharper in case of higher order elements, moreover, the distribution of the error estimator with LS seems to be evenly distributed such that $e_{h}$ and $\hat{e}_{h}$ correlate perfectly. Therefore our error estimator can maintain an accurate $h p$-adaptive refinement algorithm [13], [21].

\section{REFERENCES}

[1] R. A. Adams and J. J. Fournier. Sobolev spaces. Academic Press, Amsterdam, second edition, 2003. Pure and Applied Mathematics, Vol. 140.

[2] M. Ainsworth and A. Craig. A posteriori error estimators in the finite element method. Numer. Math., 60(4):429-463, 1992.

[3] M. Ainsworth and J. T. Oden. A posteriori error estimation in finite element analysis. Pure and Applied Mathematics. Wiley-Interscience [John Wiley \& Sons], New York, 2000.

[4] M. Ainsworth and R. Rankin. Fully computable bounds for the error in nonconforming finite element approximations of arbitrary order on triangular elements. SIAM J. Numer. Anal., 46(6):3207-3232, 2008.

[5] I. Babuška and W. C. Rheinboldt. Error estimates for adaptive finite element computations. SIAM J. Numer. Anal., 15(4):736-754, 1978.

6] R. E. Bank and A. Weiser. Some a posteriori error estimators for elliptic partial differential equations. Math. Comp., 44(170):283-301, 1985.

[7] R. E. Bank and J. Xu. Asymptotically exact a posteriori error estimators. I. Grids with superconvergence. SIAM J. Numer. Anal., 41(6):2294-2312 (electronic), 2003.

[8] R. E. Bank and J. Xu. Asymptotically exact a posteriori error estimators. II. General unstructured grids. SIAM J. Numer. Anal., 41(6):2313-2332 (electronic), 2003.

[9] S. C. Brenner and L. R. Scott. The mathematical theory of finite element methods, volume 15 of Texts in Applied Mathematics. Springer-Verlag, New York, second edition, 2002

[10] C. Carstensen. Some remarks on the history and future of averaging techniques in a posteriori finite element error analysis. ZAMM Z. Angew. Math. Mech., 84(1):3-21, 2004.

[11] C. Carstensen. A unifying theory of a posteriori finite element error control. Numer. Math., 100(4):617-637, 2005.

[12] C. Carstensen and S. Bartels. Each averaging technique yields reliable a posteriori error control in FEM on unstructured grids. I. Low order conforming, nonconforming, and mixed FEM. Math. Comp., 71(239):945-969 (electronic), 2002.

[13] L. Demkowicz. Computing with hp-adaptive finite elements. Vol. 2. Chapman \& Hall/CRC Applied Mathematics and Nonlinear Science Series. Chapman \& Hall/CRC, Boca Raton, FL, 2007. Frontiers: Three dimensional elliptic and Maxwell problems with applications.

[14] A. Ern and J.-L. Guermond. Theory and practice of finite elements. Springer-Verlag, New York, 2004.

[15] D. Harutyunyan, F. Izsák, J. J. W. van der Vegt, and M. A. Botchev. Adaptive finite element techniques for the Maxwell equations using implicit a posteriori error estimates. Comput. Methods Appl. Mech. Engrg., 197(17-18):1620-1638, 2008.

[16] F. Izsák, D. Harutyunyan, and J. J. W. van der Vegt. Implicit a posteriori error estimates for the Maxwell equations. Math. Comp., 77(263):1355-1386, 2008.

[17] S. Korotov, P. Neittaanmäki, and S. Repin. A posteriori error estimation of goal-oriented quantities by the superconvergence patch recovery. J. Numer. Math., 11(1):33-59, 2003.

[18] P. Ladevèze and D. Leguillon. Error estimate procedure in the finite element method and applications. SIAM J. Numer. Anal., 20(3):485-509, 1983.

[19] W. McLean. Strongly elliptic systems and boundary integral equations. Cambridge University Press, Cambridge, 2000.

[20] J. Schöberl. A posteriori error estimates for Maxwell equations. Math. Comp., 77:633-649, 2008.

[21] C. Schwab. p- and hp-finite element methods. Numerical Mathematics and Scientific Computation. The Clarendon Press Oxford University Press, New York, 1998. Theory and applications in solid and fluid mechanics.

[22] R. Verfürth. A Review of A Posteriori Error Estimation and Adaptive Mesh-Refinement Techniques. Advances in Numerical Mathematics. Wiley - Teubner, Chichester - Stuttgart, 1996.

[23] R. Verfürth. A posteriori error estimators for convection-diffusion equations. Numer. Math., 
80(4):641-663, 1998.

[24] M. Vohralík. A posteriori error estimates for lowest-order mixed finite element discretizations of convection-diffusion-reaction equations. SIAM J. Numer. Anal., 45(4):1570-1599 (electronic), 2007.

[25] L. B. Wahlbin. Superconvergence in Galerkin finite element methods, volume 1605 of Lecture Notes in Mathematics. Springer-Verlag, Berlin, 1995.

[26] O. C. Zienkiewicz and J. Z. Zhu. The superconvergent patch recovery and a posteriori error estimates. I. The recovery technique. Internat. J. Numer. Methods Engrg., 33(7):1331$1364,1992$.

[27] O. C. Zienkiewicz and J. Z. Zhu. The superconvergent patch recovery and a posteriori error estimates. II. Error estimates and adaptivity. Internat. J. Numer. Methods Engrg., 33(7):1365-1382, 1992. 


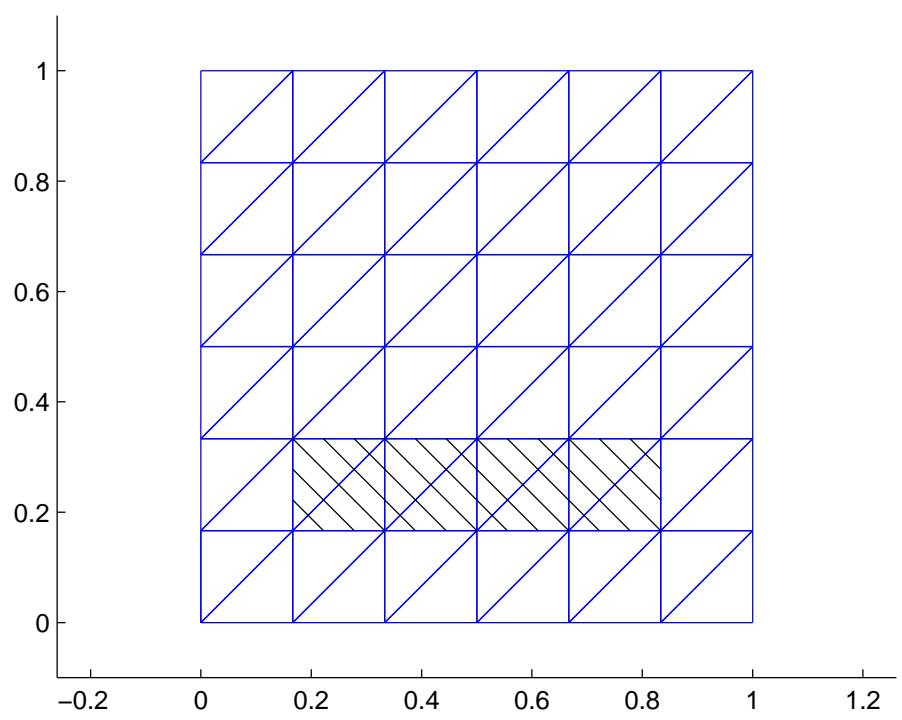

FIG. 5.1. Uniform mesh for the computations, with the shaded elements in the 2nd row, where the comparison of the local accuracy has been performed, see Figures 5.2 - 5.4.
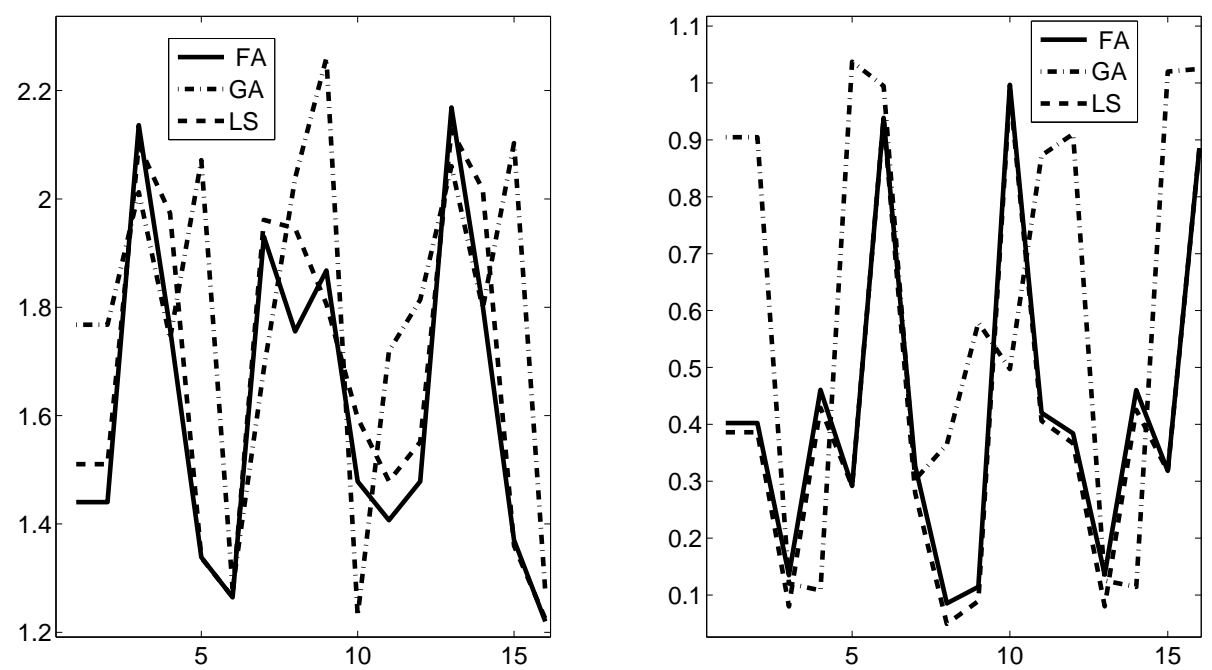

FIG. 5.2. Local accuracy of the implicit error estimation technique using the gradient recovery operators FA, GA and LS in Test Case 1 with $u_{1} \in P_{1}$. Left: error of the approximation of $L_{2}$ norm in the Neumann boundary data (see (5.4)) on the depicted elements in Fig. 5.1. Right: approximation error for the error in $\mathrm{H}_{1}$ norm (see (5.5)) on the depicted elements in Fig. 5.1. 

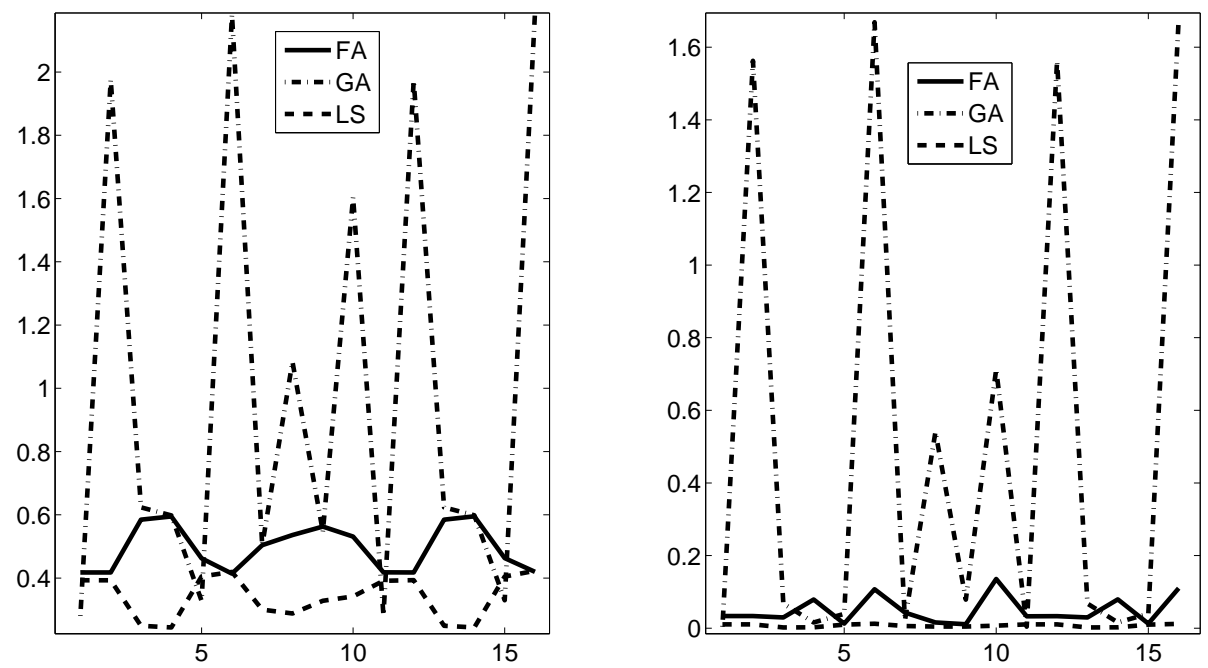

FIG. 5.3. Local accuracy of the implicit error estimation technique using the gradient recovery operators FA, GA and LS in Test Case 1 with $u_{1} \in P_{2}$. Left: error of the approximation of $L_{2}$ norm in the Neumann boundary data (see (5.4)) on the depicted elements in Fig. 5.1. Right: approximation error for the error in $H_{1}$ norm (see (5.5)) on the depicted elements in Fig. 5.1.
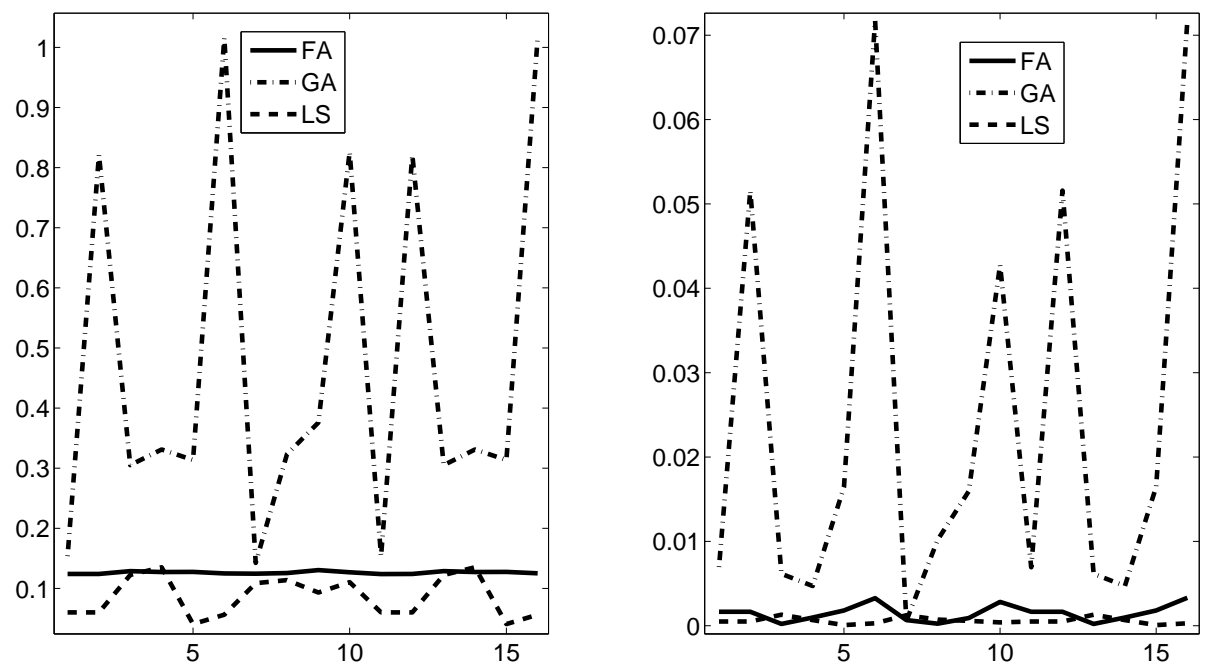

FIG. 5.4. Local accuracy of the implicit error estimation technique using the gradient recovery operators FA, GA and LS in Test Case 1 with $u_{1} \in P_{3}$. Left: error of the approximation of $L_{2}$ norm in the Neumann boundary data (see (5.4)) on the depicted elements in Fig. 5.1. Right: approximation error for the error in $H^{1}$ norm (see (5.5)) on the depicted elements in Fig. 5.1. 